\title{
Calcific tendinitis of the shoulder in the Korean population: demographics and its relation with coexisting rotator cuff tear
}

\author{
Yon-Sik Yoo ${ }^{1}$, Jin-Young Park ${ }^{2}$, Myung-sun Kim ${ }^{3}$, Nam-Su Cho ${ }^{4}$, Yong-Beom Lee ${ }^{5}$, Seung-Hyun Cho ${ }^{6}$, \\ Kyoung Jin Park ${ }^{7}$, Chul-Hyun Cho ${ }^{8}$, Bong Gun Lee ${ }^{9}$, Dong Joo Shin ${ }^{10}$, Han-Hoon Kim ${ }^{11}$, Tae Kang Lim ${ }^{11}$ \\ ${ }^{1}$ Department of Orthopedic Surgery, Hallym University Dongtan Sacred Heart Hospital, Hwaseong, Korea \\ ${ }^{2}$ Global Center for Shoulder, Elbow and Sports at Neon Orthopaedic Clinic, Seoul, Korea \\ ${ }^{3}$ Department of Orthopaedics, Chonnam National University College of Medicine, Gwangju, Korea \\ ${ }^{4}$ Department of Orthopedic Surgery, Kyung Hee University Hospital at Gangdong, Seoul, Korea \\ ${ }^{5}$ Department of Orthopaedic Surgery, Hallym University Sacred Heart Hospital, Anyang, Korea \\ ${ }^{6}$ Seoul Metropolitan Seonam Hospital, Seoul, Korea \\ ${ }^{7}$ Goodsam Orthopaedic Clinic, Cheongju, Korea \\ ${ }^{8}$ Department of Orthopedic Surgery, Keimyung University Dongsan Medical Center, Keimyung University School of Medicine, Daegu, Korea \\ ${ }^{9}$ Department of Orthopaedics, Hanyang University College of Medicine, Seoul, Korea \\ ${ }^{10}$ Department of Orthopaedics, Daegu Fatima Hospital, Daegu, Korea \\ ${ }^{11}$ Department of Orthopedic Surgery, Nowon Eulji Medical Center, Eulji University School of Medicine, Seoul, Korea
}

\begin{abstract}
Background: To evaluate the demographics, clinical and radiographic features of calcific tendinitis of the shoulder in the Korean population, specifically focusing on the incidence of coexisting rotator cuff tear.

Methods: Between October 2014 and January 2015, we performed a prospective multicenter study with 506 patients from 11 training hospitals in Korea. We collected data of demographics and radiographic analysis based on simple radiographs, clinical assessments based on visual analog scale (VAS) and the American Shoulder Elbow Surgeons (ASES) score, and treatment modalities that are used currently. We also evaluated coexisting rotator cuff tear by ultrasonography (US) or magnetic resonance imaging (MRI) images.

Results: There were 402 female patients (79\%) with mean age of 55 years (range, 31-87 years). Mean duration of symptoms was 16 months. Mean size of calcific materials was $11.4 \mathrm{~mm}$ (range, 0-35 mm). Mean value of VAS and ASES scores were 6.5 (range, 1-10) and 47 (range, 8-95), respectively. Of 383 patients (76\%), 59 (15\%) had rotator cuff tear including 15 full-thickness tears on US or MRI. Patients with rotator cuff tears were significantly associated with older age, recurrent symptoms, menstrual disorders in females, and having undergone calcification removal surgery and rotator cuff repair (all $\mathrm{p}<0.05$ ).

Conclusions: This study reported demographic, radiographic, and clinical features of calcific tendinitis of the shoulder in Korean population, which were not different from those of Western population. Coexisting rotator cuff tear was found with $15 \%$ incidence in this large series, suggesting that further radiographic study to evaluate rotator cuff tear might be needed in some calcific tendinitis patients of older age and presenting with recurrent symptoms.
\end{abstract}

Keywords: Calcific tendinitis; Epidemiology; Rotator cuff

Received: January 14, 2020 Revised: December 4, 2020 Accepted: December 6, 2020

Correspondence to: Tae Kang Lim

Department of Orthopedic Surgery, Nowon Eulji Medical Center, Eulji University School of Medicine, 68 Hangeulbiseong-ro, Nowon-gu, Seoul 01830, Korea

Tel: +82-2-970-8036, Fax: +82-2-973-3024, E-mail: shouldertk@gmail.com, ORCID: https://orcid.org/0000-0001-8752-3987

Financial support: None.

Conflict of interest: None.

Copyright(C) 2021 Korean Shoulder and Elbow Society. All Rights Reserved.

This is an Open Access article distributed under the terms of the Creative Commons Attribution Non-Commercial License (http://creativecommons.org/licenses/by-nc/4.0/) which permits unrestricted non-commercial use, distribution, and reproduction in any medium, provided the original work is properly cited. 


\section{INTRODUCTION}

Calcific tendinitis is one of the common causes of shoulder pain and discomfort, and an incidence rate of $2.7 \%-20 \%$ has been reported [1]. Several hypotheses have been raised as to the cause of calcific tendinitis, including active cell-mediated process, rotator cuff tendon degeneration, overuse and microinjury, genetic predisposition, local metabolic or hemodynamic abnormalities, and subacromial impingements, but the exact pathophysiology remains unclear [2]. The calcific tendinitis of the shoulder is prevalent in the dominant and more frequently used arm [1]. It is highly correlated to old age, diabetes, or smoking and frequently occurs in patients who raise their arms overhead [2].

Demographic factors can be related to the pathogenesis of disease and also affect its prognosis. In calcific tendinitis, it has been reported that being female, bilateral morbidity, and having multiple calcific deposits are associated with poor prognosis in longterm clinical results [3]. Contrary to the existing belief that the treatment of calcific tendinitis is benign and self-limited, de Witte et al. [3] recently reported that pain and discomfort of the shoulder still persisted in more than $50 \%$ of patients even after 14 years of follow-up. Considering these outcomes, studies of demographic and radiographic features to determine treatment options and assess prognosis at the initial diagnosis of calcific tendinitis are clinically highly significant.

Most of the existing studies [4] on demographic factors, radiographic characteristics, long-term course, and prognostic factors of the disease are limited by small numbers of patients. Studies involving larger numbers of patients are needed. Besides, studies $[3,5]$ that included relatively large numbers of research subjects were all conducted outside the country. No studies have been reported investigating demographic factors and characteristics associated with calcific tendinitis of the shoulder in the Korean population.

One of the controversies related to calcific tendinitis of the shoulder is whether it is related to the rotator cuff tear. Since calcific tendinitis can be easily diagnosed with only a simple radiographic examination, it is common to first perform conservative treatment without first performing expensive imaging tests such as magnetic resonance imaging (MRI) [5]. In patients who do not respond to conservative treatment, however, a common clinical occurrence is that accompanying rotator cuff tear is diagnosed when additional imaging tests are performed. These clinical experiences occur in patients with calcific tendinitis of the shoulder diagnosed by simple radiographic examination. The question remains as to whether there is a need to diagnose early the presence of rotator cuff tear. Indeed, according to a study by
Jim et al. [4], rotator cuff rupture was reported in 22 of 81 patients with calcified tendinitis. However, this study is limited in the number of patients, and because it is a study performed through simple radiographs of arthrography after administration of a contrast agent, diagnostic accuracy is limited. In addition to this study, the presence of rotator cuff tear in calcified tendinitis of the shoulder has been reported rarely.

Therefore, the purpose of this study was to investigate the demographic, clinical, and radiological factors of calcific tendinitis of the shoulder joint in a large number of Koreans and to investigate the prevalence of rotator cuff tear associated with calcific tendinitis.

\section{METHODS}

This study was approved by the Institutional Review Board of Nowon Eulji Medical Center (IRB No. EMCIRB 19-126), and informed consent was waived due to retrospective nature of this study.

\section{Materials}

From November 2014 to January 2015, a retrospective study was conducted on 506 patients diagnosed with calcific tendinitis of the shoulder at 11 major training hospitals nationwide. In this study, the inclusion criteria were patients that visited the hospital for shoulder pain and a simple radiographic examination with observed calcium deposits around the shoulder. Patients with infections or previous fracture or surgery in the same area of the shoulder joint were excluded from the study.

\section{Demographic and Clinical Evaluation}

Demographic characteristics of patient age, sex, dominant arm, bilateral arm, trauma history, occupation, regular exercise, hobbies using shoulders, and smoking were investigated. Occupations were categorized into housework, labor, office work, and others. Labor and office work were classified according to whether arms were used in vocational activities. Regular exercise was defined as occurring at least twice a week (ex. swimming or jogging, etc.). Smoking was defined as only current smokers. Diabetes, thyroid disorder, and rheumatoid arthritis were investigated as comorbid diseases, and the history of menopause and obstetrics and gynecological disease was investigated in females. Diseases that affect estrogen metabolism such as endometriosis, ovarian cysts, polycystic ovary syndrome, and infertility treatment history were investigated. At the first visit, the duration of symptoms, the presence of nocturnal pain, visual analog scale (VAS), and American Shoulder Elbow Surgeons (ASES) scores 
were measured for clinical evaluation. The investigation classified treatments into nonsurgical such as extracorporeal shock wave treatment or surgical.

\section{Radiological Evaluation}

The location of calcium was evaluated in the standard radiographs of anteroposterior, axial and supraspinatus outlet views. Calcium located in the anterior of the scapular spine and over the greater tuberosity was particularly noted as calcium deposit in the supraspinatus tendon. Deposits in the posterior of the scapular spine and the greater tuberosity were considered as in the infraspinatus tendon. Calcium originating from the subscapularis was determined as the case in front of the lesser tuberosity in the outlet and axial view. The size of calcium deposit was measured on picture archiving and communicating system (PACS) and its characteristic was classified by Gartner method [6] (Fig. 1). Furthermore, ultrasound or MRI was performed to investigate the presence or absence of accompanying rotator cuff tear.

\section{Statistical Analysis}

Based on these results, the relationship between demographic variables such as age, sex, presence of comorbid diseases, clinical symptoms, radiographic findings, and surgical treatment was statistically analyzed. The clinical relationship between symptoms and surgery was statistically analyzed, particularly the relation between rotator cuff tear and demographic factors. A chisquare test was performed for nominal variable analysis, and an independent $\mathrm{t}$-test was performed for continuous variable analysis. Cases where the p-value was less than 0.05 were judged to have statistical significance.

\section{RESULTS}

\section{Demographic Factor}

Of the total 506 patients, there were 402 females (79\%) and 104 males (21\%). Of the total 506 patients, there were 402 females (79\%) and 104 males (21\%) and the average age was 55 years (range, $31-87$ years). By age group, the 50s had the highest incidence at $45 \%$, followed by $23 \%$ in 60 s, $21 \%$ in 40 s, $6 \%$ in 70 s, and $5 \%$ in 30 s (Fig. 2). Among the total patients, 404 (79\%) were confirmed as being affected in the dominant arm, and 98 (19\%) cases were bilaterally affected at the time of diagnosis. There were 64 patients $(12.6 \%)$ who had a history of trauma before the onset of symptoms.

As for the occupation, unemployed, including housekeeping, was the most common, with 187 (37\%), followed by laborers with 108 (21\%), office workers with 94 (19\%), and others with 87 (17\%). One hundred and six patients (21\%) answered that they

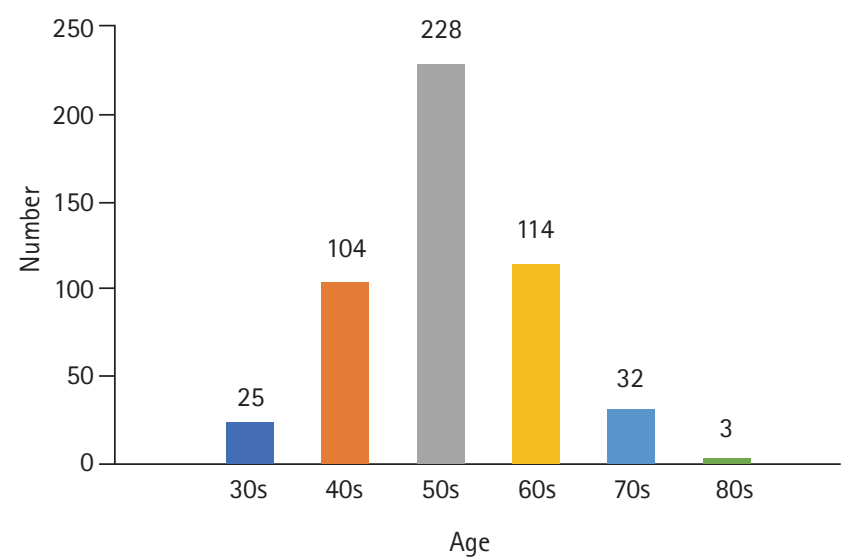

Fig. 2. Distribution of calcific tendinitis by age group. The $50 \mathrm{~s}$ had the highest incidence at $45 \%, 60$ s were $23 \%, 40$ s at $21 \%, 70$ s at $6 \%$, and 30 s at $5 \%$ by age group.
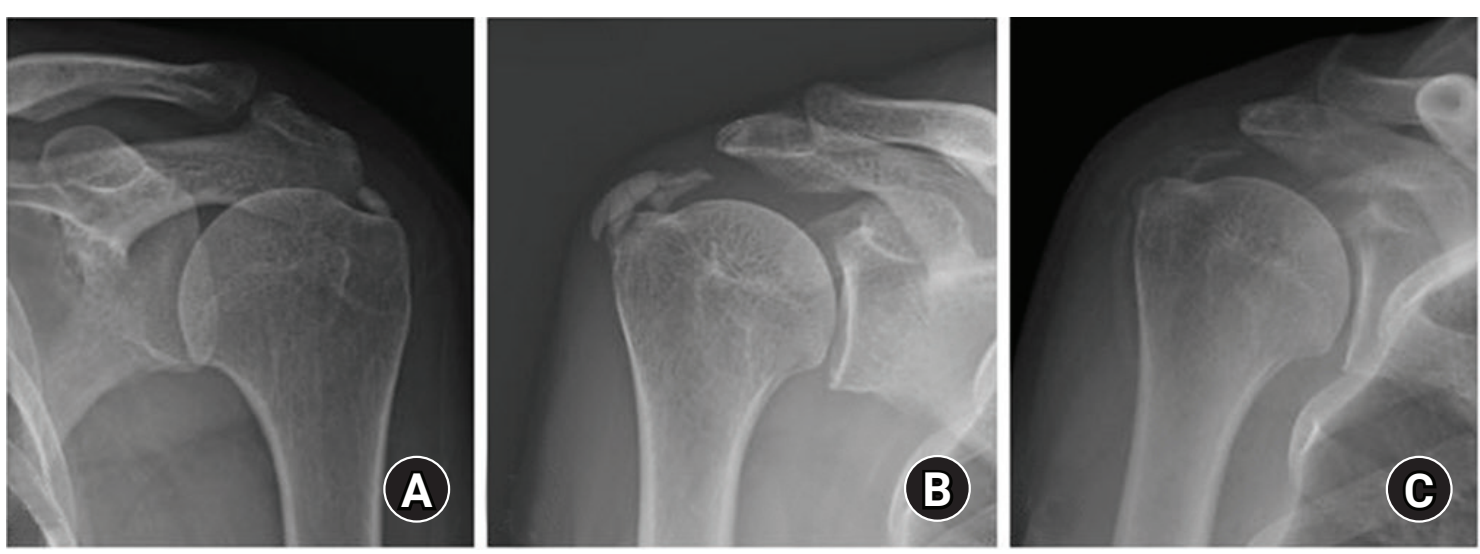

Fig. 1. Gartner classification of calcific tendinitis. (A) Gartner type 1 has a clear, uniform, and dense shape. (B) Gartner type 2 has a clear boundary but is uneven inside or a uniform inside but an uneven boundary. (C) Gartner type 3, with no borders, transparent or cloud-like. 
were exercising regularly, and 77 (15\%) were exercising using their shoulders. The current smokers included 42 (8\%). As for systemic and medical diseases, diabetes was present in 62 (12\%), thyroid disease in $32(6 \%)$, and rheumatic disease in $13(3 \%)$. Among 377 female patients, 179 (47\%) had achieved menopause, and $39(10 \%)$ had a history of obstetric diseases (Table 1).

Table 1. Demographics and clinical outcomes

\begin{tabular}{|c|c|}
\hline Variable & Value \\
\hline Age (yr) & $55(31-87)$ \\
\hline Female sex & $402(79)$ \\
\hline Dominant side & $404(79)$ \\
\hline Bilateral & $98(19)$ \\
\hline Symptom duration (mo) & $16(0-180)$ \\
\hline Recurrent symptom & $102(20)$ \\
\hline Trauma history & $64(13)$ \\
\hline Night pain & $358(71)$ \\
\hline \multicolumn{2}{|l|}{ Occupation } \\
\hline Housework & $187(39)$ \\
\hline Manual labor & $108(21)$ \\
\hline Office work & $94(19)$ \\
\hline Other & $87(17)$ \\
\hline Regular exercise & $106(21)$ \\
\hline Exercise using shoulder & $77(15)$ \\
\hline Smoking & $42(8)$ \\
\hline Diabetes & $62(12)$ \\
\hline Thyroid disease & $32(6)$ \\
\hline Rheumatoid disease & $13(3)$ \\
\hline Menopause & $179(47)$ \\
\hline Obstetric disease & $39(10)$ \\
\hline Body mass index $\left(\mathrm{kg} / \mathrm{m}^{2}\right)$ & $23.6(16.42-41.67)$ \\
\hline Size of calcification (mm) & $11.4(0-35)$ \\
\hline \multicolumn{2}{|l|}{ Gartner classification } \\
\hline Type I & $171(34)$ \\
\hline Type II & $177(35)$ \\
\hline Type III & $120(24)$ \\
\hline Coexisting rotator cuff tear & $59(15)$ \\
\hline Visual analog scale score & $6.5(1-10)$ \\
\hline American Shoulder Elbow Surgeons score & $47(8-95)$ \\
\hline Operation & $30(6)$ \\
\hline
\end{tabular}

Values are presented as mean (range) or number (\%).

Table 2. Clinical characteristics by sex

\begin{tabular}{lccc}
\hline Variable & $\begin{array}{c}\text { Male } \\
(\mathrm{n}=104)\end{array}$ & $\begin{array}{c}\text { Female } \\
(\mathrm{n}=401)\end{array}$ & p-value \\
\hline Age $(\mathrm{yr})$ & $56.7 \pm 10.4$ & $55.1 \pm 8.9$ & 0.124 \\
Recurrent symptom & $22(21)$ & $102(25)$ & 0.443 \\
Night pain & $63(61)$ & $295(73)$ & 0.015 \\
Trauma history & $13(13)$ & $50(13)$ & 0.993 \\
Visual analogue scale score & $6.2 \pm 1.9$ & $6.6 \pm 2.0$ & 0.067 \\
Size of calcification $(\mathrm{mm})$ & $11.4 \pm 7.2$ & $11.4 \pm 6.1$ & 0.972 \\
\hline
\end{tabular}

Values are presented as mean \pm standard deviation or number (\%).
When comparing clinical characteristics according to gender, the frequency of night pain in females was statistically significantly higher ( $73 \%$ for women and $61 \%$ for men, $\mathrm{p}=0.015)$. Other demographic and clinical characteristic differences between men and women were not observed (Table 2).

\section{Clinical Features}

The average symptom duration was 16 months (range, 1-180 months), the average pain score (VAS) at the time of visit was 6.6 (range, 1-10), and the ASES score was 47 (range, 8-95). Previously, 102 (20\%) were diagnosed with calcific tendinitis and had recurrence of symptoms after a symptom-free period. Sleep disturbance due to night pain was observed in 358 (70.7\%). The most common treatment for patients was the use of oral analgesics (93\%), steroid injection therapy (53\%), physical therapy (42\%), extracorporeal shock wave therapy (16\%), and surgical arthroscopic calcium removal and rotator cuff repair (6\%).

\section{Radiological Results}

The average size of calcium deposit was $11.6 \mathrm{~mm}$ (range, 0.9-35 $\mathrm{mm}$ ) on simple radiographic examination. The location of occurrence was supraspinatus: 322 cases (64\%), Infraspinatus 125 cases (25\%), subscapularis 79 cases (16\%), and Teres Minor 5 cases (1\%). According to Gartner classification [6], there were 171 cases of type 1 (34\%), 177 cases of type 2 (35\%), and 120 cases of type 3 (24\%). Ultrasound or MRI was performed in 383 out of 506 cases (76\%), of which 59 (15\%) were diagnosed with rotator cuff tear. Partial-thickness tear was more common with 44 cases (11\%), but patients with full-thickness tear (Fig. 3) were also observed in 15 cases (4\%). (Fig. 4)
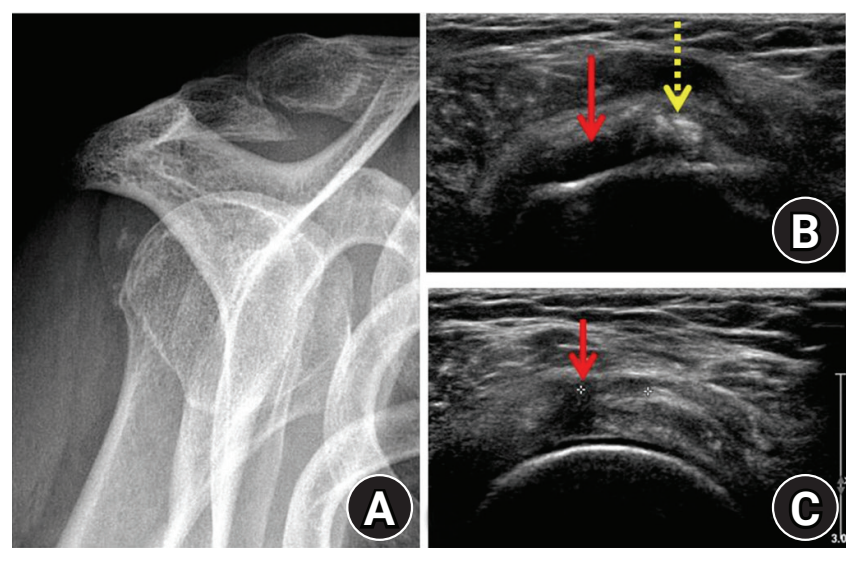

Fig. 3. A 59-year-old female patient with calcific tendinitis with rotator cuff tear. (A) Radiation examination of the shoulder joint exit showed calcium in the inferior tendon. $(\mathrm{B}, \mathrm{C})$ Ultrasound examination showed rotator cuff tear (solid arrows) and calcium deposition at the same time (dotted arrow). 
When comparing calcific tendinitis patients with rotator cuff tear against those without rotator cuff tear on ultrasound or MRI, the average age of patients with rotator cuff tear was 58 years, significantly higher than the 55 year average age of patients without $(p=0.025)$. In addition, the associated frequency of rotator cuff tear in patients with recurring symptoms (41\%) was significantly higher $(\mathrm{p}=0.012)$ than in the other group $(23 \%)$. The frequency $(83 \%)$ was significantly higher than that of the other group $(68 \%)(p=0.042)$. In patients who had menopause, the associated frequency of rotator cuff tears (50\%) was significantly higher than in the group without menopause $(35 \%, \mathrm{p}=0.014)$. The associated frequency of rotator cuff tear in the surgical group (33\%) was also significantly higher than in the nonoperative group $(10 \%, \mathrm{p}<0.001)$ (Table 3$)$.

\section{DISCUSSION}

This study is a multicenter epidemiologic investigation of the demographics, imaging, and clinical features of calcific tendinitis in

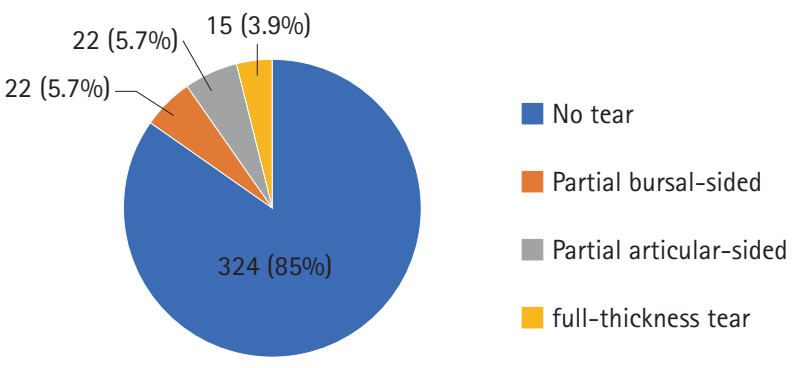

Fig. 4. Rotator cuff tear associated with calcific tendinitis. Rotator cuff tear accompanied in 15\% of cases: partial rupture of the mucous sac was present in $5.7 \%$, partial rupture of the joint in $5.7 \%$, and $3.9 \%$ showed full rupture.
Koreans. The most important finding was that $15 \%$ of patients with calcific tendinitis had a rotator cuff tear. The average symptom age of calcific tendinitis was 55 years, and it tended to be more prevalent in females. About $71 \%$ of patients had a high rate of night pain. Among treatment modalities, oral analgesics (93\%) were the most common, followed by steroid injection therapy (53\%).

According to Harvie et al.'s study [7] on the relationship between the natural course of calcific tendinitis and endocrine diseases, the prevalence of endocrine diseases such as hypothyroidism was high in patients with calcific tendinitis. The proportion of female patients with calcific tendinitis was high, suggesting that estrogen might play a role in the pathogenesis. In this study's results, the prevalence of females was at $79 \%$, diabetes was confirmed in $12 \%$ of patients, and thyroid disease was also confirmed in $6 \%$ of patients. Among female patients with calcific tendinitis, previous studies reported that menstrual irregularities and hysterectomy rates were higher than those of the general population [7] and that finding corresponded to a high proportion of menstrual irregularities (47\%) and obstetric diseases (10\%) found in this study.

According to the results of a long-term follow-up study by de Witte et al. [3], long-term prognosis was poor when calcific tendinitis invaded the dominant arm or bilateral arms. It is more common for calcific tendinitis to invade the dominant arm [1] because the rotator cuff contracts during the lifting motion, making it hemodynamically susceptible to local ischemia [2]. In this study, it was confirmed that the dominant arm invading patients accounted for a large proportion (79\%) of cases, and it was more frequent in houseworkers and laborers than in office workers. As such, a patient's dominant arm activities in occupation and hobbies is related to the cause of the disease and the prognosis, and

Table 3. Clinical characteristics comparing groups with and without coexisting rotator cuff tear

\begin{tabular}{lccc}
\hline Variable & No rotator cuff tear $(\mathrm{n}=324)$ & Coexisting rotator cuff tear $(\mathrm{n}=59)$ & $\mathrm{p}$-value \\
\hline Age $(\mathrm{yr})$ & $55.2 \pm 8.9$ & $58.0 \pm 8.6$ & 0.025 \\
Female sex & $241(74)$ & $42(71)$ & 0.157 \\
Recurrent symptom & $76(23)$ & $24(41)$ & 0.012 \\
Night pain & $250(68)$ & $48(83)$ & 0.042 \\
Trauma history & $48(15)$ & $10(17)$ & 0.297 \\
Diabetes & $30(9)$ & $2(6)$ & 0.748 \\
Thyroid diseases & $26(8)$ & $4(7)$ & 0.556 \\
Menopause & $113(35)$ & $29(50)$ & 0.014 \\
Obstetric disease & $30(9)$ & $7(12)$ & 0.087 \\
Rheumatoid disease & $12(4)$ & $2(3)$ & 0.66 \\
Size of calcification $(\mathrm{mm})$ & $11.1 \pm 6.0$ & $12.3 \pm 7.2$ & 0.332 \\
Operation & $31(10)$ & $19(32)$ & $<0.001$ \\
\hline
\end{tabular}

Values are presented as mean \pm standard deviation or number (\%). 
so it must be carefully checked before treatment.

According to previous studies, the radiological size and Gartner classification of calcium cannot accurately predict patients' prognosis. Still, radiographic improvement (reduction in size or improvement of Gartner classification) over time was associated with good clinical outcomes [3]. As a complication of calcific tendinitis, the rotator cuff tear can occur before or during surgery. Of these, $23 \%$ of patients reported that rotator cuff tear was also present. Hsu et al. [8] also reported that calcific tendinitis and rotator cuff tear occurred together in $28 \%$ of patients on shoulder angiography and that calcium deposits were rather small in patients with rotator cuff tear. Gotoh et al. [9] reported that calcific tendinitis could progress and become a rotator cuff tear. The rotator cuff tear repeats inflammation and regeneration, and calcium deposits reveal a small amount of calcium on the radiograph [10]. In this study, concomitant rotator cuff tear was confirmed in $15 \%$ of patients who underwent ultrasound or MRI examination. The patient group with rotator cuff tear was older and had more recurrence of symptoms and menstrual irregularities than the group without the rotator cuff tear. Rotator cuff tear was also significantly more likely in women. The rotator cuff tear was associated with a high frequency of $32 \%$ of the group that underwent surgery perhaps due to the more active surgical treatment. In the case of calcific tendinitis, conservative treatment is generally performed first. The prognosis is generally good, but worsens in patients with persistent symptoms, with recurring symptoms, old age, or patients with menstrual irregularities [11]. In some cases, rotator cuff tear can coexist with calcific tendinitis of the shoulder. Therefore, an active imaging test should be performed, and treatment should be performed with the accompanying rotator cuff tear in mind.

This study has several limitations. First, the frequency of calcific tendinitis occurrence relative to the population was unknown. There was no control group, so the risk factors for inducing calcific tendinitis were unknown. Second, as a multicenter study, several different observers measured calcium deposit size in a simple imaging test. There is a possibility that a difference in reliability among testers may occur. In addition, the diagnosis of rotator cuff tear on MRI cannot exclude the reliability factor among examiners. Ultrasound in particular was performed by several examiners leading to a limitation in that the examination was not necessarily consistent. The accuracy may be different for each institution. Third, whether the rotator cuff was ruptured due to degenerative changes or a burst of calcium, it was impossible to clarify the pathophysiology, i.e., whether calcium was produced due to inflammation or regeneration.

This study reported demographic, radiographic and clinical features of calcific tendinitis of the shoulder in the Korean population, which were not different from those of Western populations. Coexisting rotator cuff tear was found with 15\% incidence in this large series, suggesting that further radiographic study to evaluate rotator cuff tear might be needed in some calcific tendinitis patients of older age and with recurrent symptoms.

\section{ORCID}

Tae Kang Lim https://orcid.org/0000-0001-8752-3987

\section{REFERENCES}

1. Speed CA, Hazleman BL. Calcific tendinitis of the shoulder. N Engl J Med 1999;340:1582-4.

2. Sansone V, Maiorano E, Galluzzo A, Pascale V. Calcific tendinopathy of the shoulder: clinical perspectives into the mechanisms, pathogenesis, and treatment. Orthop Res Rev 2018; 10:63-72.

3. de Witte PB, van Adrichem RA, Selten JW, Nagels J, Reijnierse M, Nelissen RG. Radiological and clinical predictors of longterm outcome in rotator cuff calcific tendinitis. Eur Radiol 2016;26:3401-11.

4. Jim YF, Hsu HC, Chang CY, Wu JJ, Chang T. Coexistence of calcific tendinitis and rotator cuff tear: an arthrographic study. Skeletal Radiol 1993;22:183-5.

5. Suzuki K, Potts A, Anakwenze O, Singh A. Calcific tendinitis of the rotator cuff: management options. J Am Acad Orthop Surg 2014;22:707-17.

6. Gärtner J, Simons B. Analysis of calcific deposits in calcifying tendinitis. Clin Orthop Relat Res 1990;(254):111-20.

7. Harvie P, Pollard TC, Carr AJ. Calcific tendinitis: natural history and association with endocrine disorders. J Shoulder Elbow Surg 2007;16:169-73.

8. Hsu HC, Wu JJ, Jim YF, Chang CY, Lo WH, Yang DJ. Calcific tendinitis and rotator cuff tearing: a clinical and radiographic study. J Shoulder Elbow Surg 1994;3:159-64.

9. Gotoh M, Higuchi F, Suzuki R, Yamanaka K. Progression from calcifying tendinitis to rotator cuff tear. Skeletal Radiol 2003;32: 86-9.

10. Merolla G, Bhat MG, Paladini P, Porcellini G. Complications of calcific tendinitis of the shoulder: a concise review. J Orthop Traumatol 2015;16:175-83.

11. Bazzocchi A, Pelotti P, Serraino S, et al. Ultrasound imaging-guided percutaneous treatment of rotator cuff calcific tendinitis: success in short-term outcome. Br J Radiol 2016;89:20150407. 